\title{
Hair cortisol concentration in a population without hypothalamic-pituitary-adrenal axis disorders
}

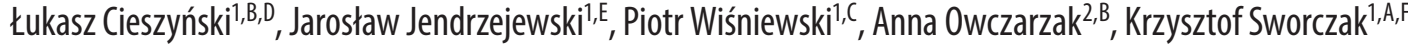 \\ ${ }^{1}$ Department of Endocrinology and Internal Medicine, Medical University of Gdańsk, Poland \\ ${ }^{2}$ Department of Clinical Nutrition and Dietetics, Medical University of Gdańsk, Poland \\ A - research concept and design; $B$ - collection and/or assembly of data; $C$ - data analysis and interpretation; \\ $D$ - writing the article; $E$ - critical revision of the article; $F$ - final approval of the article
}

\section{Address for correspondence}

Łukasz Cieszyński

E-mail: lukaszdoc@wp.pl

Funding sources

None declared

Conflict of interest

None declared

Received on November 22, 2017

Reviewed on December 9, 2017

Accepted on April 18, 2018

Published online on December 5, 2018

\begin{abstract}
Background. Measuring hair cortisol seems to be a good alternative to laboratory tests used thus far in routine endocrine diagnostics, primarily because it is independent of the circadian rhythm of cortisol. Due to the average hair growth of $1 \mathrm{~cm}$ per month, the results are related to the average blood cortisol levels over the previous weeks, months or years (depending on the length of the hair sample).
\end{abstract}

Objectives. The aim of this study is an attempt to apply hair cortisol concentration (HCC) measurements to clinical endocrine diagnostics, based on reference cortisol concentrations in the blood in a population without disorders of the hypothalamic-pituitary-adrenal axis (HPA).

Material and methods. In the final selection process, 44 patients were enrolled in the study, all with negative interviews regarding disorders of the HPA and with reference levels of cortisol concentration obtained in routine laboratory tests. In the pre-analytic phase, we used $1 \mathrm{~cm}$ proximal hair strands cut from the posterior vertex area of the head, followed by the incubation of a $20 \mathrm{mg}$ hair sample in methanol. The final cortisol measurement was done using an enzyme-linked immunosorbent assay (ELISA).

Results. The results of HCC ranged from $2 \mathrm{pg} / \mathrm{mg}$ up to $51.63 \mathrm{pg} / \mathrm{mg}$. The diurnal decrease in cortisol levels was significantly lower in females than in males $(p=0.031)$, but we do not consider that difference to be clinically significant. The difference in the $\mathrm{HCC}$ between males and females was not statistically significant $(p=0.767)$. The linear regression coefficient for age was not statistically significant $(p=0.847)$. Neither the regression coefficients for gender nor the gender and age interactions were statistically significant $(p=0.815)$.

Conclusions. Hair cortisol concentration measurement, unlike other endocrinological tests, gives information about the cortisol concentration in the long-term perspective. The results obtained in this study may be used as a reference for further research aimed at determining normal values of HCC.

Key words: measurements, validity, hair cortisol, reference

Cite as

Cieszyński Ł, Jendrzejewski J, Wiśniewski P, Owczarzak A, Sworczak K. Hair cortisol concentration in a population without hypothalamic-pituitary-adrenal axis disorders. Adv Clin Exp Med. 2019;28(3):369-373. doi:10.17219/acem/90038

DOI

10.17219/acem/90038

\section{Copyright}

Copyright by Author(s)

This is an article distributed under the terms of the

Creative Commons Attribution Non-Commercial License

(http://creativecommons.org/licenses/by-nc-nd/4.0/) 


\section{Introduction}

The aim of this study was an attempt to apply measurements of cortisol concentrations in hair to clinical endocrine diagnosis, based on reference cortisol concentrations in the blood in a population with no disorders of the hypothalamic-pituitary-adrenal axis (HPA).

Routine endocrine diagnostics for patients with HPA axis disorders entail a series of blood, urine and saliva tests. These include daily serum cortisol, urine free cortisol (UFC), saliva cortisol, and several hormonal dynamic tests using stimulation, such as the corticotropin-releasing hormone (CRH) stimulation test, or inhibition test, e.g., the low/high dose dexamethasone suppression test. The numerous methods of cortisol assessment arise from the necessity of collecting reliable results that correlate with a clinical picture. Their interpretation is often impeded due to variations in the method of determination, environmental factors, physiological variations in cortisol levels, drugs/xenobiotics taken, and pathological conditions secondarily affecting the HPA axis. For this reason, there is no single test that will produce a credible result, and therefore, at least a few tests must be performed to provide a basis for a proper diagnosis. ${ }^{1-3}$

Publications related to hair cortisol concentrations (HCC) mainly refer to non-primary endocrine disorders, including depressive-anxiety disorders, stressful events, shift work, physical exertion, alcohol abuse, smoking, chronic illnesses, or doping control. ${ }^{4-12}$ Among the most relevant endocrine disorders in the context of HCC assays are Cushing's syndrome, Cushing's subclinical syndrome, Addison's disease, secondary adrenal insufficiency, and cortisol/hydrocortisone substitution..$^{13-20}$

Measurement of HCC seems to be a good alternative to the laboratory tests used thus far in diagnosing endocrine disorders, primarily due to its independence from the circadian rhythm of cortisol. Given the average hair growth of $1 \mathrm{~cm}$ per month, the results are related to average blood cortisol levels over the past weeks, months or years, depending on the length of the hair sample. ${ }^{21-23}$

The presence of cortisol in hair, as well as other steroid hormones, is most probably a result of the passive diffusion of free cortisol from blood to the hair matrix during its formation in hair follicles. Another possible explanation for the presence of cortisol in hair is its presence in sweat and sebum. Hence, its concentration may depend on additional factors such as gender (stimulation of sweat and sebum production by androgens), age (slower hair growth with age), hair dyeing (possible flushing out of cortisol by chemicals, interference with HCC assays), physical activity, hygienic habits (influence of hair washing frequency), or exposure to sunlight. ${ }^{11,24-31}$ Additional benefits of HCC assessment are its non-invasive sample collection procedure and the possibility of sample storage at room temperature for a long period of time without prior processing.
Hair cortisol concentration measurement is most often based on immunological methods, such as the enzymelinked immunosorbent assay (ELISA) or liquid chromatography tandem mass spectrometry (LC-MS/MS). ${ }^{27,32-37}$ Measurement of HCC in humans was initiated by J.S. Raul in $2004 .^{36}$ The population analyzed in his study consisted of 44 volunteers recruited on the basis of a questionnaire. The HCC measurement was done using chromatography, and the amount of cortisol in the samples ranged from $5 \mathrm{pg} / \mathrm{mg}$ to $91 \mathrm{pg} / \mathrm{mg}$. Since the first report, a number of publications analyzing the HCC measurement have involved different populations of subjects recruited on the basis of demographic and anthropometric factors. ${ }^{24}$ The reliability of HCC measurement is limited due to the heterogeneity of the populations analyzed, the various methodologies of the assays, comparative assessment without initial laboratory diagnostics regarding blood cortisol concentrations, and the lack of commonly accepted HCC reference values. An attempt to establish reference values of HCC in healthy subjects who have also been evaluated for cortisol levels using standard laboratory tests remains ongoing.

\section{Material and methods}

We included 88 patients who were hospitalized in the Department of Endocrinology and Internal Medicine at the Medical University of Gdańsk, Poland, for the evaluation of hormone levels during the course of an incidental adrenal tumor. All participants signed a written informed consent to participate in the study. The study was approved by the Bioethical Committee of the Medical University of Gdańsk. Enrollment in the project was carried out in 2016.

The routine diagnostic protocol consisted of measurement of morning plasma adrenocorticotropic hormone (ACTH) (ACTH8am), morning plasma cortisol (MPC8am), evening plasma cortisol (EPC8pm), 24-hour urinary free cortisol excretion (UFC24h), and the $1 \mathrm{mg}$ overnight dexamethasone suppression test (1mgONDST). These measurements were performed at the Central Laboratory of the University Hospital in Gdańsk. Patients taking glucocorticosteroids during the preceding year and those with conditions likely to affect the results of the tests - in particular depression, alcoholism, acute/chronic inflammatory disease, and primary or secondary HPA disorders - were excluded from the study. In the final selection process, $44 \mathrm{pa}-$ tients were enrolled in the study, all with reference levels of ACTH8am (reference: <46 pg/mL), MPC8am (reference: 101-535 nmol/L), EPC8pm (reference: 79-478 nmol/L), UFC24h (reference: $12-486 \mathrm{nmol} / 24 \mathrm{~h}$ ), a regular diurnal decrease of cortisol (DDC; reference: $\mathrm{EPC}<\mathrm{MPC}$ more than 50\%), and proper suppression of cortisol after $1 \mathrm{mgONDST}$ (reference: $<50 \mathrm{nmol} / \mathrm{L}$ ).

A hair sample was collected in the examination room from the posterior vertex area of the head, using a sterile 
Table 1. Characteristics of the population. Data is presented as mean \pm standard deviation (SD) and median \pm interquartile range (IQR)

\begin{tabular}{|c|c|c|c|c|}
\hline Variable & Total & Female & Male & $\mathrm{p}$-value \\
\hline Number & 44 & 31 & 13 & n/a \\
\hline Age [years] & $63.3 \pm 10.3$ & $63.6 \pm 8.8$ & $62.5 \pm 13.8$ & 0.757 \\
\hline Morning plasma cortisol [nmol/L] & $338.0 \pm 113.8$ & $323.7 \pm 109.4$ & $375.0 \pm 120.3$ & 0.175 \\
\hline Evening plasma cortisol [nmol/L] & $111.1 \pm 46.8$ & $115.2 \pm 46.8$ & $101.4 \pm 47.1$ & 0.379 \\
\hline Diurnal decrease of cortisol [\%] & $-65.5 \pm 11.9$ & $-63.0 \pm 10.6$ & $-71.4 \pm 13.1$ & 0.031 \\
\hline Urine free cortisol [nmol/24 h] & $204.3 \pm 130.2$ & $201.7 \pm 127.9$ & $210.7 \pm 140.7$ & 0.837 \\
\hline ACTH $[p g / m L]$ & $\begin{array}{c}29.95 \pm 20.01 \\
(22.8 \pm 15.7)\end{array}$ & $\begin{array}{l}27.67 \pm 19.11 \\
(22.0 \pm 14.4)\end{array}$ & $\begin{array}{c}35.39 \pm 21.81 \\
(26.8 \pm 19.1)\end{array}$ & 0.095 \\
\hline 1mgONDST [nmol/L] & $\begin{array}{c}32.27 \pm 6.07 \\
(28.0 \pm 8.0)\end{array}$ & $\begin{array}{c}33.23 \pm 6.61 \\
(30.0 \pm 9.0)\end{array}$ & $\begin{array}{c}30.00 \pm 3.83 \\
(28.0 \pm 3.0)\end{array}$ & 0.1374 \\
\hline
\end{tabular}

1mgODST - 1 mg overnight dexamethasone suppression test; ACTH - adrenocorticotropic hormone; n/a - not applicable.

scalpel to cut an approximate quantity of 100-200 hair strands as close as possible to the scalp. The samples obtained were stored in dry envelopes until further analysis. Next, $1 \mathrm{~cm}$ segments of the hair strands were cut away near the follicles, and following weighing, exactly $20 \mathrm{mg}$ of the sample was used in the study. The preparation of the hair samples was performed at the Department of Clinical Nutrition and Dietetics at the Medical University of Gdańsk. First, the hair samples were placed in $10 \mathrm{~mL}$ plastic tubes and flushed with $2 \mathrm{~mL}$ of methanol. Next, they were transferred to $5 \mathrm{~mL}$ tubes and incubated at $50^{\circ} \mathrm{C}$ in $1 \mathrm{~mL}$ of methanol for $24 \mathrm{~h}$. The extract was then transferred to $3 \mathrm{~mL}$ tubes, after which it was evaporated. Finally, the residue was dissolved in $250 \mu \mathrm{L}$ of phosphate-buffered saline (PBS) by incubation for $1 \mathrm{~h}$.

The final HCC measurement was done at the Central Laboratory of the University Hospital in Gdańsk using an ELISA Cortisol Assay (IBL International GmbH, Hamburg, Germany; catalog No. RE 52611). The assay is reported to demonstrate cross-reactivity with prednisolone (30\%), 11-desoxy-cortisol (7\%), cortisone (4.2\%), prednisone (2.5\%), corticosterone (1.4\%), and less than $1 \%$ with other tested substances. The limit of detection and functional sensitivity are calculated to be $0.005 \mathrm{ug} / \mathrm{dL}$ and $0.030 \mathrm{ug} / \mathrm{dL}$, respectively. A detailed description of the assay is available on the manufacturer's website (http://www.ibl-international. com/en/cortisol-saliva-elisa). For each sample, the HCC was measured twice, and the average value was used in the statistical analysis.

Standard descriptive statistics were prepared. Conformity to a normal distribution was assessed using a histogram analysis. Some of the dependent variables underwent a logarithmic transformation before they were included in the parametric analysis. The arithmetic mean of the $2 \mathrm{HCC}$ assessments was analyzed. The results of HCC are presented as $\mathrm{pg} / \mathrm{mg}$ according to the cortisol concentration in the analyzed volume of the solution $(250 \mu \mathrm{L})$ based on the amount of incubated hair $(20 \mathrm{mg})$. Comparisons in subgroups were evaluated using the t-test or the MannWhitney U test. A multivariate regression (general linear) analysis was used to evaluate the relationship between age and HCC. The regression model also included gender and analyzed the interaction between age and gender. P-values $<0.05$ were considered statistically significant. All the calculations were performed using the STATA Statistical Package v. 13.1 (StataCorp LLC, College Station, USA).

\section{Results}

The size of the population, demographic data and the parameters of the pituitary-adrenal axis that were evaluated are presented in Table 1 . The diurnal decrease in cortisol levels was significantly lower in females than in males $(\mathrm{p}=0.031)$. However, we consider that difference not to be clinically significant. There were no statistically significant differences between other variables.

The results of HCC ranged from $2 \mathrm{pg} / \mathrm{mg}$ up to $51.63 \mathrm{pg} / \mathrm{mg}$. The difference in HCC between males and females was not statistically significant $(\mathrm{p}=0.767)$. The detailed characteristics of the HCC results are presented in Table 2. We also analyzed the relationship between age and HCC. The results are shown in Fig. 1. The linear regression coefficient for age was not statistically significant ( $\mathrm{p}=0.847$ ). The regression coefficients for gender and for gender and age interactions were also not statistically significant $(\mathrm{p}=0.815)$.

Table 2. Hair cortisol concentration (HCC) results [pg/mg]

\begin{tabular}{|l|c|c|c|}
\hline \multicolumn{1}{|c|}{ Variable } & $\begin{array}{c}\text { Total } \\
(\mathrm{n}=44)\end{array}$ & Female $(\mathrm{n}=31)$ & $\begin{array}{c}\text { Male } \\
(\mathrm{n}=13)\end{array}$ \\
\hline Mean & 7.17 & 7.74 & 5.82 \\
\hline SD & 10.40 & 12.05 & 4.63 \\
\hline Median & 3.38 & 3.25 & 3.63 \\
\hline IQR & 2.97 & 2.56 & 6.19 \\
\hline Minimum & 2.00 & 2.00 & 2.13 \\
\hline Maximum & 51.63 & 51.63 & 17.06 \\
\hline
\end{tabular}

SD - standard deviation; IQR - interquartile range. 
A. $p=0.733$

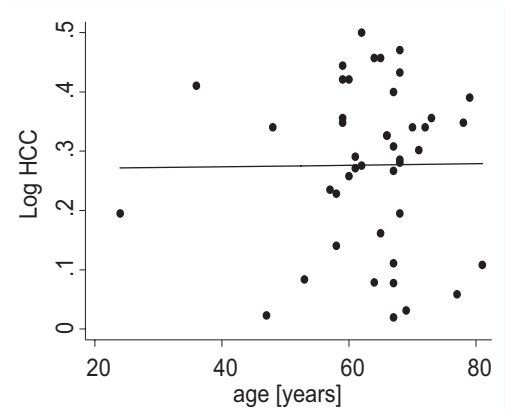

B. $p=0.926$

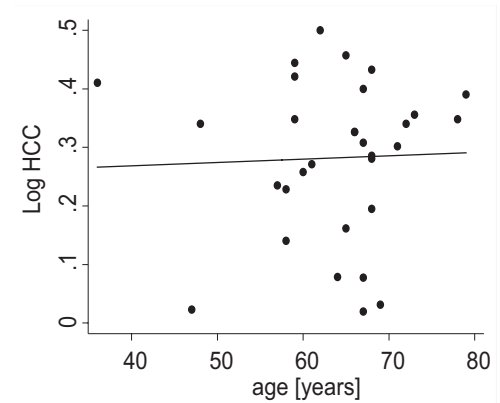

C. $p=0.680$

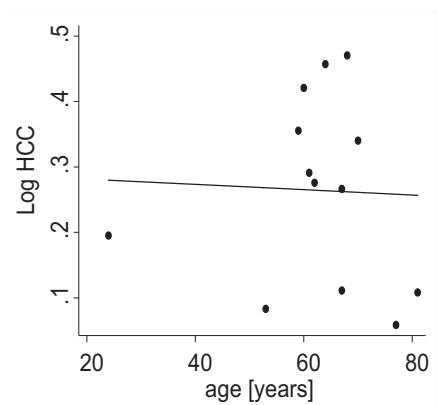

Fig. 1. Association between hair cortisol concentration (HCC) and age

A - all; B - females; C - males.

\section{Discussion}

Hair cortisol concentration assessment is a multistep process, starting with proper hair sampling, followed by extraction of cortisol at the pre-analytical stage and concluding with final laboratory evaluation using one of the reference methods. Each of these steps can have a significant impact on the final result. Hence, the various phases of HCC assessment have been subjected to evaluation in numerous studies. In our study, the number of collected hair strands from a given individual was largely a result of a compromise between the need to obtain as much material as possible and esthetic issues, especially among the female population. In most studies, the amount of hair analyzed ranges from $2.5 \mathrm{mg}$ to $150 \mathrm{mg}$, with the most widely used amount being 10-20 mg. $6,733,34,38$ The optimal site for hair sampling is the vertex posterior area of the head; possible individual variation in $\mathrm{HCC}$ can be as high as $24 \%$, depending on the site of collection, as demonstrated by drug and xenobiotic studies. ${ }^{39}$ Incubation of a total hair sample (1-centimeter fragments) paradoxically results in higher HCC than milled hair, as it has been shown in 1 work describing HCC methodology. ${ }^{40}$ Extraction of cortisol using a methanol solution is one of the most commonly used methods. ${ }^{4,24,41}$ Analyses of various methods of HCC determination indicate the superiority of ELISA in terms of the sensitivity of cortisol measurement. ${ }^{40,42}$ This assay is very useful due to its widespread availability as well as its ease of implementation. The correlations between HCC measurements with ELISA and LC-MS/MS in interlaboratory comparisons have been found to be strong. ${ }^{42}$

The essential problem in HCC analysis is to determine the degree of cortisol extraction from hair samples. Generally, we assumed close to $100 \%$ cortisol recovery by methanol extraction, which might not be fully true based on the latest reports. One publication has demonstrated higher extraction efficiency by methanol and acetone over methanol alone ${ }^{40}$ However, most published works used cortisol extraction by methanol as a reference method.

Unlike previous studies, our work focused on the deep characteristics of the study population. The recruited volunteers may be regarded as a reference group because of their extensive screening for regular levels of cortisol. Thanks to the recruitment protocol, the impact of anthropometric and demographic parameters possibly affecting the HPA axis during hair collection (e.g., gender, body weight, hair color, race) has largely been excluded. We believe that higher maximal HCC in females $(\mathrm{n}=31, \mathrm{HCC}=51.63 \mathrm{pg} / \mathrm{mg})$ vs males $(\mathrm{n}=13$, $\mathrm{HCC}=17.06 \mathrm{pg} / \mathrm{mg}$ ) may possibly be due to the small male population tested and may not be related to other factors. Most studies exclude gender as a potential confounding factor in adult participants. ${ }^{13,27,33,36}$ The issues outlined in Material and methods section meant that only 44 patients out of 88 met the final inclusion criteria based on the study protocol (incorrect DDC and 1mgONDST were the most common reasons for exclusion). An interview that excluded the enrollment of patients with primary or secondary HPA disorders in the period $>1$ year prior to sampling further strengthens our results since analyzed hair samples reflected an average cortisol concentration of past month only.

\section{Conclusions}

The results of the study indicate the possibility of using HCC measurement in all subjects under the condition of receiving a sufficient hair sample. Cortisol extraction using a methanol medium is easy to perform with basic laboratory equipment. Commercial ELISA assays have high sensitivity and specificity with a very low cortisol detection threshold.

The study was conducted on a homogeneous group of participants who did not display corticotropic axis disorders. The HCC ranged from $2 \mathrm{pg} / \mathrm{mg}$ to $51.63 \mathrm{pg} / \mathrm{mg}$. The results obtained in the present work may be used as a reference for further research.

Hair cortisol measurement may be a new diagnostic tool in clinical practice. Further research on HCC may contribute to improvement of the diagnosis and treatment of patients with HPA axis diseases. Hair cortisol concentration measurement, unlike other endocrinological tests, provides information about cortisol concentration in the patient's long-term history. 


\section{References}

1. Isidori AM, Kaltsas GA, Pozza C, et al. The ectopic adrenocorticotropin syndrome: Clinical features, diagnosis, management, and longterm follow-up. J Clin Endocrinol Metab. 2006;91(2):371-377.

2. Nieman LK, Biller BM, Findling JW, et al. The diagnosis of Cushing's syndrome: An endocrine society clinical practice guideline. J Clin Endocrinol Metab. 2008;93(5):1526-1540.

3. Cieszyński L, Berendt-Obołonczyk M, Szulc M, Sworczak K. Cushing's syndrome due to ectopic ACTH secretion. Endokrynol Pol. 2016;67(4): 458-464.

4. Stalder T, Kirschbaum C. Analysis of cortisol in hair: State of the art and future directions. Brain Behav Immun. 2012;26(7):1019-1029.

5. D'Anna-Hernandez KL, Ross RG, Natvig CL, et al. Hair cortisol levels as a retrospective marker of hypothalamic-pituitary axis activity throughout pregnancy: Comparison to salivary cortisol. Physiol Behav. 2011;104(2):348-353.

6. Vanaelst B, Michels N, De Vriendt T, et al. Cortisone in hair of elementary school girls and its relationship with childhood stress. Eur J Pediatr. 2013;172(6):843-846.

7. Kamps AW, Molenmaker M, Kemperman R, et al. Children with asthma have significantly lower long-term cortisol levels in their scalp hair than healthy children. Acta Paediatr. 2014;103(9):957-961.

8. Kirschbaum C, Tietze A, Skoluda N, et al. Hair as a retrospective calendar of cortisol production: Increased cortisol incorporation into hair in the third trimester of pregnancy. Psychoneuroendocrinology. 2009;34(1):32-37.

9. Stalder T, Kirschbaum C, Heinze K, et al. Use of hair cortisol analysis to detect hypercortisolism during active drinking phases in alcoholdependent individuals. Biol Psychol. 2010;85(3):357-360.

10. Veldhorst MA, Noppe $G$, Jongejan $M H$, et al. Increased scalp hair cortisol concentrations in obese children. J Clin Endocrinol Metab. 2014;99(1):285-290.

11. Dettenborn L, Muhtz C, Skoluda N, et al. Introducing a novel method to assess cumulative steroid concentrations: Increased hair cortisol concentrations over 6 months in medicated patients with depression. Stress. 2012;15(3):348-353.

12. Vives AH, De Angel V, Papadopoulos A, et al. The relationship between cortisol, stress and psychiatric illness: New insights using hair analysis. J Psychiatr Res. 2015;70:38-49.

13. Thomson S, Koren G, Fraser LA, et al. Hair analysis provides a historical record of cortisol levels in Cushing's syndrome. Exp Clin Endocrinol Diabetes. 2010;118(2):133-138.

14. Staufenbiel SM, Andela CD, Manenschijn L, et al. Increased hair cortisol concentrations and BMI in patients with pituitary-adrenal disease on hydrocortisone replacement. J Clin Endocrinol Metab. 2015;100(6):2456-2462.

15. Manenschijn L, Koper JW, van den Akker ELT, et al. A novel tool in the diagnosis and follow-up of (cyclic) Cushing's syndrome: Measurement of long-term cortisol in scalp hair. J Clin Endocrinol Metab. 2012;97(10):E1836-E1843.

16. Gow R, Koren G, Rieder M, et al. Hair cortisol content in patients with adrenal insufficiency on hydrocortisone replacement therapy. Clin Endocrinol (Oxf). 2011;74(6):687-693.

17. Wester VL, Reincke M, Koper JW, et al. Scalp hair cortisol for diagnosis of Cushing's syndrome. Eur J Endocrinol. 2017;176(6):695-703.

18. Manenschijn L, Quinkler M, van Rossum EFC. Hair cortisol measurement in mitotane-treated adrenocortical cancer patients. Horm Metab Res. 2014;46(4):299-304.

19. Noppe G, Rossum EFC, Vliegenthart J, et al. Elevated hair cortisol concentrations in children with adrenal insufficiency on hydrocortisone replacement therapy. Clin Endocrinol (Oxf). 2014;81(6):820-825.
20. Hodes A, Lodish MB, Tirosh A, et al. Hair cortisol in the evaluation of Cushing syndrome. Endocrine. 2017;56(1):164-174.

21. Wennig R. Potential problems with the interpretation of hair analysis results. Forensic Sci Int. 2000;107(1):5-12.

22. Balíková M. Hair analysis for drug abuse. Plausibility of interpretation. Biomed Pap Med Fac Univ Palacky Olomouc Czech Repub. 2005; 149(2):199-207.

23. LeBeau MA, Montgomery MA, Brewer JD. The role of variations in growth rate and sample collection on interpreting results of segmental analyses of hair. Forensic Sci Int. 2011;210(1):110-116.

24. Staufenbiel SM, Penninx BW, Rijke YB, et al. Determinants of hair cortisol and hair cortisone concentrations in adults. Psychoneuroendocrinology. 2015;60:182-194.

25. Feller S, Vigl M, Bergmann MM, et al. Predictors of hair cortisol concentrations in older adults. Psychoneuroendocrinology. 2014;39:132-140.

26. Stalder T, Steudte S, Miller R, et al. Intraindividual stability of hair cortisol concentrations. Psychoneuroendocrinology. 2012;37(5):602-610.

27. Manenschijn L, Koper JW, Lamberts SW, et al. Evaluation of a method to measure long term cortisol levels. Steroids. 2011;76(10):1032-1036.

28. Grass J, Kirschbaum C, Miller R, et al. Sweat-inducing physiological challenges do not result in acute changes in hair cortisol concentrations. Psychoneuroendocrinology. 2015;53:108-116.

29. Wester VL, van der Wulp NR, Koper JW, et al. Hair cortisol and cortisone are decreased by natural sunlight. Psychoneuroendocrinology. 2016;72:94-96.

30. Hoffman MC, Karban LV, Benitez P, et al. Chemical processing and shampooing impact cortisol measured in human hair. Clin Invest Med. 2014;37(4):E252-E257.

31. Hamel AF, Meyer JS, Henchey E, et al. Effects of shampoo and water washing on hair cortisol concentrations. Clin Chim Acta. 2011;412(3): 382-385.

32. Gow R, Thomson S, Rieder M, et al. An assessment of cortisol analysis in hair and its clinical applications. Forensic Sci Int. 2010;196(1):32-37.

33. Gao W, Xie Q, Jin J, et al. HPLC-FLU detection of cortisol distribution in human hair. Clin Biochem. 2010;43(7):677-682.

34. Noppe G, Rijke YB, Dorst K, et al. LC-MS/MS-based method for longterm steroid profiling in human scalp hair. Clin Endocrinol (Oxf). 2015; 83(2):162-166.

35. Bévalot F, Gaillard Y, Lhermitte MA, et al. Analysis of corticosteroids in hair by liquid chromatography-electrospray ionization mass spectrometry. J Chromatogr B Biomed Sci Appl. 2000;740(2):227-236.

36. Raul JS, Cirimele V, Ludes B, et al. Detection of physiological concentrations of cortisol and cortisone in human hair. Clin Biochem. 2004;37(12):1105-1111.

37. Cirimele V, Kintz P, Dumestre V, et al. Identification of ten corticosteroids in human hair by liquid chromatography-ionspray mass spectrometry. Forensic Sci Int. 2000;107(1):381-388.

38. Sauvé B, Koren G, Walsh G, et al. Measurement of cortisol in human hair as a biomarker of systemic exposure. Clin Invest Med. 2007;30(5): 183-191.

39. Tanada N, Kashimura S, Kageura M, et al. Utility of caffeine analysis for forensic hair discrimination. Nihon Hoigaku Zasshi. 1998;52: 233-237.

40. Slominski R, Rovnaghi CR, Anand KJ. Methodological considerations for hair cortisol measurements in children. Ther Drug Monit. 2015; 37(6):812-820.

41. Wester VL, Rossum EF. Clinical applications of cortisol measurements in hair. Eur J Endocrinol. 2015;173(4):M1-M10.

42. Russell E, Kirschbaum C, Laudenslager ML, et al. Toward standardization of hair cortisol measurement: Results of the first international interlaboratory round robin. Ther Drug Monit. 2015;37(1):71-75. 